\title{
Sociología como disciplina de nivel medio en Argentina, Brasil y Uruguay ${ }^{1}$
}

\author{
Sociology as a highschool discipline in Argentina, Brazil and Uruguay
}

\author{
Paula Cuestas \\ paula.cuestas90@gmail.com \\ Centro Interdisciplinario de Metodología de las \\ Ciencias Sociales - Instituto de Investigaciones \\ en Humanidades y Ciencias Sociales- Facultad de \\ Humanidades y Ciencias de la Educación, Argentina
}

Recepción: 02 Diciembre 2019

Aprobación: 15 Julio 2020

Publicación: 02 Agosto 2020

Cita sugerida: Cuestas, P. (2020). Sociología como disciplina de nivel medio en Argentina, Brasil y Uruguay. Cuestiones de Sociología, 23, e104. https://

doi.org/10.24215/23468904e104
Resumen: Este artículo busca describir las formas en que la Sociología se constituyó como una disciplina específica en el nivel medio en Argentina, Brasil y Uruguay, sin ahondar en las diferencias regionales al interior de cada territorio nacional. Para ello se analizarán los contextos particulares de su surgimiento en cada país, así como los modos en que actualmente se imparte la asignatura en las escuelas a partir de un breve análisis de distintos recursos aúlicos como manuales, normativas como los diseños curriculares o las últimas leyes nacionales educativas, de cada caso. Luego de una breve introducción, se recupera cada una de las experiencias en particular para, a continuación, hacer un análisis comparativo a partir de lo presentado. El trabajo muestra cómo la sociología, en tanto asignatura de nivel medio, ha sido asociada a distintas tradiciones o influida por otras disciplinas, en cada país y en el contexto particular de su surgimiento. Nos guía la pregunta por el sentido de esta materia en las aulas escolares y su potencial para promover el "pensamiento crítico".

Palabras clave: Enseñanza de Sociología, Nivel medio, Argentina, Brasil, Uruguay.

Abstract: This article describes how Sociology was constituted as a specific discipline in high school in Argentina, Brazil and Uruguay, wit hout developing the regional differences with in each national territory. In order tot his, the particular contexts of Sociology's emergence in each country will be analyzed, as well as theways in which this subject is currently taught in schools, based on a brief analysis of different schoolresourcessuch as manuals, regulationssuch as curricular designs or the latest national education allaws, in each case. After a briefintroduction, eachof the experiences isrecovered to make a comparative analysis based on whatwas presented. The papershows how Sociology, as a high school subject, has been associated with different traditions or influenced by other disciplines, in each country and in the context ofits emergence. The question is guided by the meaning of this subject in school classrooms and its potential to promote critical thin king.

Keywords: Teaching Sociology, High School, Argentina, Brazil, Uruguay. 
“...Si uno es tan puntilloso sobre el carácter cientifico de la sociología, es porque se trata de una disciplina que incomoda (...) Porque revela cosas ocultas y a veces reprimidas..."

(Bourdieu, 1990,pp. 61-62)

\section{A Modo DE INTRODUCCIÓN}

Cuando se hace el ejercicio de preguntarle a un grupo de estudiantes avanzados/as del Profesorado en sociología para qué enseñar esta asignatura en la escuela secundaria, las respuestas remiten con énfasis al carácter crítico, desnaturalizador y problematizador de esta disciplina. ${ }^{2}$ Más allá de esta reflexión común que emerge entre estudiantes y también académicos/as, tal vez resulte preciso preguntarnos cómo se construye ese imaginario en torno a la disciplina, particularmente en su conformación como asignatura del nivel medio en las escuelas. El presente trabajo se motoriza a partir de esa inquietud y para ello se propone analizar los contextos particulares del surgimiento de la materia en el nivel medio en tres países latinoamericanos: Argentina, Brasil y Uruguay. ${ }^{3}$ Asimismo, se describirán los modos en que actualmente se imparte la asignatura en las escuelas a partir de una breve presentación de distintos recursos aúlicos y/o normativas como los diseños curriculares, manuales o las últimas leyes nacionales educativas, de cada caso. El enfoque comparativo se encuentra justificado por el interés en relevar distintas experiencias y en contrastar los diferentes modos en que la sociología fue pensada desde sus orígenes como disciplina. No se trata de una motivación solo cognoscitiva, sino que también se parte de considerar la importancia de generar estudios comparativos en el campo de la educación en un momento en que "las profundas transformaciones del mundo contemporáneo plantean nuevos temas y nuevos problemas a la definición de la política educativa”(Piovani y Krawczyk, 2017, p. 835).De cualquier modo, vale la pena destacar que plantear una comparación de unidades complejas, como son los territorios nacionales, puede resultar engañoso. En ese sentido, el presente trabajo no desconoce las profundas diferencias regionales que se presentan en cada caso (especialmente, al interior de los extensos territorios de Argentina y Brasil).Sin embargo, la limitación de los materiales de referencia disponibles obtura la posibilidad de un análisis pormenorizado de las diferencias regionales al interior de cada país. Es por ello que en este trabajo me referiré a los casos nacionales, considerando cada país como un todo, pero sin perder de vista esta imprescindible consideración.

Cabe resaltar, de acuerdo con el diagnóstico de Pereyra y Pontremoli (2014), que el interés por el estudio de la sociología se ha centrado especialmente en el nivel universitario y de posgrado, y que las indagaciones en torno a su surgimiento y desarrollo en el nivel medio son aún escasas. Asimismo, estos autores reflexionan, recuperando los aportes de De Cesare (2006), respecto de la distancia que suele encontrarse entre las preocupaciones de las/os docentes que se desempeñan en el nivel secundario con las discusiones del mundo universitario y académico. En ese sentido, es interesante prestar atención al carácter específico que adopta la sociología cuando se presenta como asignatura escolar. Aquí no se trata de una enseñanza con un "contenido vocacional" (pensándolo en contraste con quienes ya están estudiando esta carrera) sino de promover una forma de generar ciudadanas/os más activas/os y comprometidas/os con su sociedad (De Cesare, 2006) o de promover una forma de lo que Pipkin (2009) denomina "pensamiento social" (que se evidencia en las respuestas de los estudiantes del profesorado). De acuerdo con esta autora, esta noción designa una forma de pensamiento que busca complejizar y problematizar la realidad en una apuesta por una práctica transformadora, generadora de una conciencia crítica que en la sociología se encuentra presente desde sus inicios, en las tradiciones sociológicas propuestas desde "los clásicos" (Pipkin, 2009).

Veamos entonces cómo es que esta disciplina surge en los distintos países a analizar y de qué forma esa propuesta sociológica se inscribe o no en una línea por promover otros modos de pensar el mundo y la realidad que nos rodea. 


\section{ESCENARIO LATINOAMERICANO}

\section{I.Argentina}

Comencemos por el caso propio. La "Sociología" como asignatura obligatoria en el sistema medio argentino tiene una historia de muy pocos años. El primer punto a mencionar es que, como ya se ha destacado, al interior del país el escenario no es homogéneo. El sistema educativo está fuertemente territorializado, lo que otorga un margen de autonomía muy amplio para que cada provincia determine su currícula a partir de los lineamientos básicos que desde el Estado nacional se bajen al respecto. Es por ello que la enseñanza de la sociología en el nivel medio no es de carácter extendido ni obligatorio a todas las orientaciones en todo el territorio argentino. En general, cuando es parte del plan de estudios, se presenta como asignatura de carácter obligatorio en el último o penúltimo año de la secundaria para las orientaciones en Ciencias Sociales.

En este subapartado, me centraré especialmente en la experiencia de esta asignatura en la provincia de Buenos Aires. Siguiendo a Molinari (2009), la misma recién adquiere carácter de obligatoria a partir de las reformas educativas la década de 1990, estableciéndose como un espacio curricular para las orientaciones de Humanidades y Ciencias Sociales, con tres horas semanales en el último año. Antes de eso, existieron algunas experiencias excepcionales en colegios dependiente de Universidades y algunos colegios privados (Pereyra y Pontremoli, 2014) pero sin un carácter sistemático, ni masivo.

Actualmente, en el territorio de la provincia de Buenos Aires "Sociología" es una materia obligatoria para ciertas orientaciones (Ciencias Sociales y Educación Física) y se dicta en el quinto año del Ciclo Superior de la Escuela Secundaria con una carga horaria de tres horas semanales. El Diseño Curricular (DC) de esta asignatura se encuentra vigente desde el año 2011 (Actis et al., 2018). ${ }^{4}$ En su presentación, se establece la importancia de este espacio curricular en la escolarización de las/os estudiantes ya que se espera de la sociología una contribución a la formación de sujetos críticos, que problematicen los saberes del "sentido común", a través de herramientas teóricas y conceptuales que propicien la construcción de una mirada desnaturalizadora de la realidad social (Argentina. Ministerio de Educación, 2011). En vistas de este propósito, el DC se centra en dos tipos de contenidos conceptuales: las principales tradiciones sociológicas y los temas sobre los cuales los autores reconocidos como "clásicos" teorizaron (Actis et al., 2018).

Los DC de otras provincias argentinas ${ }^{5}$ plantean varios puntos en común con el bonaerense para plantear sus temáticas y hacer un recorte significativo de los temas a abordar. En líneas generales, predomina una organización por ejes temáticos que focalizan en: 1) el surgimiento de la sociología como ciencia o la construcción de una mirada sociológica; 2) el objeto de estudio de la sociología: las transformaciones de la sociedad (un recorrido histórico desde las revoluciones burguesas hasta la actualidad); 3) procesos de sociabilidad y socialización; 4) la sociedad como espacio de conflictos y desigualdades, procesos de inclusión y exclusión social. En la mayor parte de los casos se sigue un cronograma temático similar. Sólo en algunos DC hay unidades temáticas que apelan al desarrollo de herramientas metodológicas. Esto es: más allá de esa introducción que recupera en líneas generales "el oficio del sociológico", es mucho menor el hincapié otorgado al aprendizaje-enseñanza de técnicas específicas de esta disciplina, en favor de una orientación teórico-conceptual y del desarrollo de una "mirada sociológica".

La sanción de la Ley de Educación Nacional (LEN) en 2006 trajo aparejadas modificaciones al escenario educativo de los años 90; estas estuvieron vinculadas especialmente a la obligatoriedad del nivel secundario (nivel en el que se dicta la asignatura que aquí estamos analizando). En los siguientes apartados se problematizará brevemente sobre los alcances y limitaciones de esta normativa, en relación con la enseñanza de la sociología en el nivel medio y sus puntos en común con las otras experiencias regionales.

Un último punto a destacar dentro de este subapartado es que, actualmente, en las aulas de las escuelas secundarias, es habitual el uso de tres manuales para el dictado de Sociología: los producidos por las editoriales 
Aula Taller, Aique y Maipue. Los dos primeros son de fácil acceso en las bibliotecas escolares de la provincia de Buenos Aires, ${ }^{6}$ ya que a partir de una política de distribución de manuales del Ministerio de Educación de la Nación llegaron a muchas escuelas del país. El último, de publicación más tardía, si bien no tiene aún el mismo impacto en los cursos escolares y en la planificación de los docentes que los primeros dos, también ha cobrado significatividad en los últimos años.

\section{II.Uruguay}

El vecino país es pionero en el establecimiento de la obligatoriedad del sistema secundario. En Uruguay, a este nivel se lo reconoce como "liceos". Desde la década del 70 el ciclo básico de "el liceo" es obligatorio, a partir de la sanción de la ley 14.101 de 1973. Lo que no es obligatorio es la enseñanza de Sociología. Al día de hoy esta asignatura sólo es de carácter obligatorio en ciertas orientaciones: Ciencias Sociales y Humanidades, con una carga horaria de $4 \mathrm{~h}$ semanales en el 2 do. año del bachillerato.

La sociología, en tanto disciplina, tuvo un desarrollo tardío y algo discontinuo en Uruguay. Las primeras experiencias se dieron con la implementación de cursos de sociología en el Instituto de Ciencias Sociales, a finales de la década de 1960, en la Facultad de Derecho y Ciencias Sociales. Más tarde esto se tradujo en la creación de la Licenciatura en Sociología, la cual fue clausurada en 1973, durante el último gobierno dictatorial uruguayo, y reabierta en 1985.

Las primeras experiencias de enseñanza de la sociología en el nivel medio también datan de la década del60, a partir del llamado "Plan Piloto" creado por la Asamblea de profesores Art. 40 (Motta de Souza Martínez, 2016; Sabatovich Fernández, 2018). Se trata de una sociología estrechamente vinculada al Derecho, "con un contenido moralizador reformando la tradición normalizadora/disciplinadora" (Sabatovich Fernández, 2018). Con el tiempo, las especificidades disciplinares se fueron haciendo más tajantes (de la mano de un mayor desarrollo de las mismas también a nivel universitario) y se fue definiendo la identidad propia de la enseñanza de la sociología. ${ }^{7}$

Las distintas transformaciones a nivel educativo que se dieron a partir de la asunción del Frente Amplio a mediados de la primera década del 2000 también trajeron como consecuencia la reformulación del DC de Sociología en el 2006 (el mismopermanece vigente hasta la fecha). En el año 2008 se aprueba en Uruguay una Ley general de Educación que trae aparejadas ciertas reformas respecto de las normativas vigentes hasta entonces, especialmente de aquellas promulgadas durante la década de 1990. En términos generales, esta ley actuó, principalmente, en garantizar un alcance y una regulación nacional respecto de programas, normativas y propuestas educativas, a través de instancias de participación y diálogo en distintas comisiones de trabajo en todos los departamentos del país. En el nivel secundario, especialmente en el nivel secundario superior que es en el cual se dicta Sociología, este cambio supuso la obligatoriedad del segundo ciclo (los últimos tres años de enseñanza media) cerrando así un proceso que se iniciaba con las reformas constitucionales de 1967 y la sanción de la ley 14.101 en 1973 . De este dato no podemos inferir linealmente que la matrícula de las/ os estudiantes que asisten a clases de Sociología en el nivel medio se haya elevado significativamente, pero sí es importante destacar la existencia de esta normativa.

"Sociología" se inscribe en lo que se llama Inspección de "Educación Social y Cívica, Derecho y Sociología”, es decir, vinculada, como en el caso argentino, a la idea de contribuir a la formación de un cierto orden social. Efectivamente, la sociología se propone tanto como un instrumento con el que afrontar y explicar la sociedad actual, así como una "ciencia crítica" capaz de cuestionar "nuestras estructuras y los efectos de su funcionamiento” (Uruguay. Ministerio de Educación, 2006, p. 2).

Los contenidos en los que se organiza la asignatura son muy similares a los de los programas argentinos: 1 ) la sociología como ciencia; 2) la sociedad; 3) estratificación, clases sociales y desigualdad; y 4) cambio social (DC Uruguay, 2006). La diferencia es que aquí el caso transversal a analizar es el uruguayo. En ese sentido, 
hay un énfasis mayor que en Argentina al estudio del escenario local. Esto puede deberse a las particularidades de esta nación, mucho más pequeña y homogénea en su extensión territorial que la Argentina.

Tal como establece el propio DC, el manual más usual que se utiliza en las aulas para enseñar sociología es el producido por Adriana Marrero (investigadora y docente de la UdelaR), Introducción a la Sociología. La primera edición es de 1998 y fue pensado especialmente para el nivel medio. Si bien su formato de presentación es menos "escolar" que los que podemos encontrar en Argentina (cargados de imágenes, gráficos, actividades para los estudiantes) el carácter simplificado de los textos, a partir del ejercicio de transposición didáctica (Chevallard, 1991) que hace la autora, permite situarlo como un texto canónico dentro de las escuelas.

\section{III.Brasil}

En primer término, cabe resaltar que el sistema educativo brasilero es algo diferente al argentino y al uruguayo en cuanto a su composición y sus objetivos. Desde sus inicios ha sido atravesado por una tradición mucho más elitista y segmentada que la que se encuentra presente en la concepción del sistema educativo argentino (Tedesco, 1993) y uruguayo (Rama, 2004).

A diferencia de otros países, como Argentina, las primeras cátedras de Sociología fueron creadas en la escuela secundaria. Ya desde finales del siglo XIX se sugirió su incorporación en escuelas cuando la sociología aún no se consolidaba como carrera Universitaria. Un dato llamativo es que mientras que el establecimiento de la sociología en el ámbito universitario se centra especialmente en los Estados y ciudades pujantes del Sur, los primeros antecedentes de enseñanza de sociología en el nivel medio fueron concebidos en poblados alejados de los grandes centros urbanos. Por ejemplo, ha habido experiencias populares en Manaos o en Aracaju, al norte de Brasil. Pese a estos casos pioneros, no fue hasta los años 20 del siglo pasado que comenzó a haber experiencias más generales de enseñanza de sociología (Oliveira, 2013).

En Brasil, como afirma Oliveira (2013), esa temprana instauración de la sociología en el currículum educativo respondía al proceso de modernización que estaba llevando adelante el país. Sin embargo, ese proceso (como casi todos los procesos históricos) no fue lineal y en la década de los años 40 se dio marcha atrás en el establecimiento de Sociología como asignatura del nivel medio. Con los años, y a partir de distintas reformas educativas, así como de luchas sindicales por su restablecimiento, la sociología pasa a ganar terreno nuevamente en la currícula educativa de estados como Río de Janeiro, Minas Gerais o San Pablo. Recientemente, en el año 2005, con la creación del Grupo de Trabalho Ensino de Sociologia y, en el 2012, con la fundación de la Associacao Brasileira de Ensino de Ciencias Sociais, se consolida esta línea de investigación. De algún modo, esta gradual reintroducción de la Sociología fue amparada por la sanción de la Ley de Directrices y Bases de la Educación Nacional (LDB) de 1996. Sin embargo, no fue hasta la década de los 2000, con el gobierno de Lula Da Silva, que la Sociología finalmente establece su carácter de obligatoriedad a partir de la ley 11.683 (Oliveira, 2013). Cabe destacar que, a diferencia del caso argentino y uruguayo, en Brasil no existen orientaciones en el nivel secundario: las/os estudiantes hacen el mismo trayecto formativo, por lo que la Sociología es una disciplina común para todas/os ellas/os de acuerdo con el plan curricular brasileño. También es preciso explicitar que la obligatoriedad del nivel medio en este país recién fue consolidada a partir de una enmienda constitucional en el año 2009 (más adelante revisaremos, brevemente, los alcances y limitaciones de esta medida).

A partir de entonces los desafíos pasan a ser otros: si Sociología ya es obligatoria, ¿Qué sociología enseñar? ¿Cómo pensar un diseño curricular único en un país tan diverso? ¿Es posible? Y más aún, ¿qué sentido tiene? (Oliveira, 2013; Santos, 2012). Para encontrar algunas respuestas a estos interrogantes, Santos (2012) analiza las directrices curriculares de 14 estados de Brasil buscando los puntos comunes que permitan pensar un "mapa común" para la disciplina. Encuentra, en consecuencia, que hay ciertas categorías que emergen con fuerza en esas directrices como sociedad, cultura, identidad, poder, Estado, clases sociales que, a pesar de 
presentar un carácter polisémico, suelen remitir a la obra de autores comunes. Asimismo, observa una fuerte impronta de disciplinas como antropología y ciencias políticas en la delimitación de los temas que se abordan en sociología. Sin embargo, existen notables diferencias dentro de ese "mapa común”, que hacen a la necesidad de seguir problematizando los sentidos de una propuesta curricular de alcance nacional.

La expansión de la sociología en Brasil trae como correlato una ampliación en el número de los profesorados en Sociología. Asimismo, se han desarrollado políticas públicas en este sentido como la distribución de libros para estudiantes en todo Brasil (a excepción del Estado de San Pablo). Refiero concretamente al Plano Nacional do Livro Didático vigente desde 2012 y cuya implementación impacta en la enseñanza de la Sociología (Oliveira, 2013). El programa supone la evaluación de libros, su compra y distribución; se trata de manuales especialmente pensados para la enseñanza secundaria. Esta política editorial ha ido llevando, desde su primera versión, a una expansión en la cantidad de proyectos que se presentan para su aprobación cada año. En ese sentido, el proceso parece apuntar a una calidad cada vez mayor en los libros que se presentan y distribuyen. Finalmente, otro aspecto que se ha fortalecido en este proceso es el de la producción académica en torno a pensar y producir conocimiento sobre la enseñanza de la sociología en la escuela secundaria. No sólo en el recorrido histórico de esta disciplina sino también en la producción de materiales y reflexiones didácticas novedosas.

\section{EL ESCENARIO LATINOAMERICANO EN DiÁLOgO}

Luego de este breve recorrido por la sociología como asignatura en el nivel medio en estos tres países, debemos llamar la atención sobre más de un aspecto.

En primer lugar, las diferencias entre la sociología en el ámbito universitario y académico y su desarrollo en el nivel medio. ${ }^{8}$ Considerando únicamente instituciones de gestión pública y universitaria, en Argentina la carrera de Sociología (licenciatura y en algunos casos profesorados ${ }^{9}$ ) se dicta en unas diez universidades (muchas de las cuales no cuentan con más de 20 años de desarrollo, ${ }^{10}$ y se concentran fuertemente en la región centro del país), en Uruguay sólo en la UdelaR en la ciudad capital del país, mientras que en Brasil su expansión es mucho más amplia. En cualquiera de los casos, lo que hay es una bifurcación entre la formación docente y la producción de conocimiento, tal como señala Marrero (2010) para pensar el caso uruguayo. Si bien el trabajo de esta autora no se centra únicamente en el proceso de enseñanza-aprendizaje de sociología, su análisis nos ofrece claves para pensar la especificidad de este proceso en la asignatura que aquí nos interesa. Lo cierto es que si bien, poco a poco, profesoras/es universitarias/os de Sociología comienzan a poblar las aulas escolares, todavía la gran mayoría de la planta docente está conformada por docentes de otras disciplinas (Historia, Psicología, Ciencias de la Educación, Psicopedagogía, Derecho y otras carreras afines). Más aún, inclusive en los casos en los que quienes enseñan esta materia son profesoras/es universitarias/os de la carrera de Sociología, la propia dinámica del mundo laboral educativo tiende a segmentar la enseñanza de los procesos de investigación. Quizás, la experiencia brasilera, con la creación del Grupo de Trabalho Ensino de Sociologia y la Associacao Brasileira de Ensino de Ciencias Sociais, pueda ser pionera en pensar de otros modos la vinculación entre investigar y enseñar (Oliveira, 2013 y 2015). Asimismo, en relación con este primer punto, cabría preguntarnos si en aquellas zonas geográficas que cuentan con espacios de formación de sociólogos/as y profesores/as en la disciplina no hay una concentración de propuestas educativas de Sociología para el nivel medio, así como el carácter que adopta esta asignatura en las aulas respecto de otras regiones donde hay una densidad menor de profesionales en esta disciplina. Pensemos, por ejemplo, el caso de la provincia de Buenos Aires en el plano local donde Sociología es obligatoria para más de una orientación del nivel secundario. En esta región la carrera de Sociología a nivel universitario se imparte tanto en la capital provincial, en la Universidad Nacional de La Plata, como en la capital nacional, en la Universidad de Buenos Aires, a sólo unos $60 \mathrm{~km}$. A ambas instituciones asisten muchas/os estudiantes del Gran Buenos Aires así como del interior 
de la provincia (y del país). Sería interesante analizar el caso de aquellas regiones más aisladas y remotas de Argentina en donde no abundan profesores/as de Sociología y la materia tiene otro peso en los programas educativos. Del mismo modo cabría preguntarnos por estas diferencias en Uruguay y Brasil. Si bien esto no es objeto de este breve trabajo, es un llamado de atención respecto de las limitaciones de una propuesta comparativa en clave nacional ingenua sobre estas posibles desigualdades regionales.

En segundo lugar, es preciso recuperar el derrotero de la Sociología académica en cada país, para luego ver qué relaciones guarda con su enseñanza en el nivel medio. Mientras que en el caso argentino y uruguayo los procesos dictatoriales interrumpieron la institucionalización de la disciplina, en Brasil, paradójicamente, el régimen que llega al poder en 1964 propicia el proceso contrario. Así, bajo el lema de la "modernización" se expandieron los programas de posgrado y los subsidios para investigación (Blois, 2015). Más allá de lo interesante que resulta en sí mismo este contraste, estos datos valen la pena pues nos evitan caer en lecturas lineales que atribuyen a todos los procesos dictatoriales y autoritarios el ir en detrimento de la enseñanza de las ciencias sociales. Ahora bien, en ese análisis no podemos olvidar el peso que el discurso modernizador tenía en los artífices de la Quinta República Brasilera y que encontraba asidero en la enseñanza de la sociología, la cual ya gozaba de este carácter desde su instauración a principios de siglo en el nivel medio. Con todo, es interesante pensar qué idea de "modernización" estaba presente en aquel paradigma y, más aún, como sostiene Canclini (1997) recuperado por Oliveira (2013), incluso considerar que lo que tuvo lugar en Brasil (como en el resto de América Latina) por aquellos años fue un proceso de "modernismo sin modernización".

Asimismo, cabe detener la mirada sobre el carácter cambiante que se le atribuyea ciertas disciplinas, en particular, y a la educación, en general. El 26 de abril de 2019, Jair Bolsonaro, actual presidente de Brasil, informó, a través de su cuenta de Twitter, la intención de desfinanciar las facultades de Sociología y Filosofía, con el fin de focalizar en áreas que "generen retorno inmediato al contribuyente, como veterinaria, ingeniería y medicina" [sic]. Bien podría pensarse que un gobierno autoritario como el de Bolsonaro, con ribetes políticos, económicos y sociales afines a los modelos dictatoriales de los años 70 (Moura de Oliveira y Veríssimo Veronese, 2019), estaría en condiciones de asumir un pensamiento similar al de aquellos respecto de estos temas, considerando a la sociología como una disciplina capaz de "renovar intelectualmente al país" (Oliveira, 2013, p. 356). Sin embargo, los contextos son otros, así como los imaginarios: como señala Oliveira (2015) la sociología que "vuelve a las aulas" a finales del siglo XX no es la misma que la amparada en el paradigma modernizador de comienzos de siglo. Si adherimos al dogma bourdeano que piensa a la sociología como una "ciencia que incomoda", entendemos el temor del primer mandatario de Brasil por frenar el avance de estas ciencias que buscan propiciar miradas desnaturalizadoras de la sociedad en la que las/os ciudadanas/os se encuentran inmersas/os. Pero ¿es efectivamente la sociología esa ciencia que busca promover el "pensamiento social" (Pipkin, 2009) y "desnaturalizar lo dado"? Retomaremos esto más adelante.

Ahora bien, volvamos la mirada sobre los contextos de surgimiento no ya de la sociología como disciplina científica y académica sino respecto de su desarrollo en el nivel medio. En primer término, cabe aclarar que es llamativa la diferencia que existe entre el caso argentino y el brasilero. En el segundo de ellos, el surgimiento de la sociología en la escuela secundaria es muy temprano, inclusive anterior a su desarrollo en Estados Unidos o países de Europa Occidental en los que la disciplina estaba mucho más extendida en su faceta académica (pensemos solo en "los clásicos" de la sociología: dos de ellos alemanes y uno francés). La especificidad del caso brasilero es casi única (Pereyra y Pontremoli, 2014) y, como ya se mencionó, anclada en un imaginario marcadamente modernizador que otorgaba a la sociología una orientación en esa dirección. De algún modo, la experiencia local sigue un camino más tradicional: el desarrollo de la disciplina en el ámbito universitario trajo aparejada su posterior inclusión en las currículas escolares (similar a lo que acontece con otras asignaturas del área de ciencias sociales).

Si pensamos el caso argentino, concretamente, podemos notar que su obligatoriedad se estableció a partir de la década de 1990 (y no en todo el territorio nacional), período en el cual el sistema educativo en su conjunto asistió a un proceso de expansión de sus matrículas como consecuencia de la implementación del 
polimodal, en algunas provincias, tornando obligatoria la escolarización, al menos durante nueve años. Este proceso terminaría de consolidarse con la obligatoriedad de la escuela secundaria y el reemplazo del polimodal por el ciclo superior de la educación secundaria a partir de la sanción de la LEN de 2006. ¿Por qué en este contexto surge la necesidad de pensar una materia como Sociología? Tanto en Argentina, como en Uruguay, el fin de los procesos dictatoriales trajo aparejado el retorno a las universidades de carreras como Sociología, Filosofía y Ciencias Políticas, que en muchos casos habían sido prohibidas. Unos años más tarde, el corolario fue la incorporación de la sociología no sólo en las aulas universitarias sino también en las escolares. En Brasil, aunque con otra trayectoria, el paulatino retorno de la sociología a las escuelas también tiene el sello de un proceso que se abre tras el fin de los regímenes dictatoriales (Oliveira, 2013). Además, resulta interesante pensar que si bien estas aperturas comienzan a darse a finales de los años 80 e inicios de los 90 cuando, por ejemplo, en Argentina empieza a hacerse más extensiva la enseñanza de la sociología, es recién en la década de los años 2000 cuando asignaturas del ámbito de las ciencias sociales logran instalarse con otro peso en las currículas escolares de algunas provincias.

Desde este enfoque, y en estos nuevos tiempos, la sociología es concebida como esa "ciencia que incomoda" en la cual estaba pensando Bourdieu (1990), al menos desde su normativa. Revisando los DC vigentes (que como vimos datan de la primera década de los 2000 e inicios de la segunda), así como los manuales a partir de los cuales se dicta como asignatura en el nivel medio, es posible rastrear allí un imaginario que la acerca a una disciplina crítica, cuestionadora, capaz de pensar otros proyectos de sociedad posibles; en un escenario latinoamericano caracterizado por gobiernos postneoliberales, progresistas y de un "neointervencionismo estatal” en materia educativa (Suasnábar y Rovelli, 2010). En ese sentido, la enseñanza de sociología en países que, como los nuestros, han tenido experiencias autoritarias y gobiernos dictatoriales con características comunes, se vuelve una necesidad. Más aún, podemos recuperar a Lahire (2006) para pensar que la enseñanza de la sociología puede (y tal vez debe) ser una disciplina para impartir desde los primeros años escolares. El autor llama a esta idea "el desarrollo de una utopía realista" (utopía incluso en el contexto francés), y se pregunta: ¿por qué no enseñar ciencias sociales (concretamente sociología y antropología) desde la escuela primaria? ¿por qué no "atacarlas" desde el principio, plantea, "siendo que en la universidad y siguiendo la mayor tradición mítica, las ciencias calificadas de 'duras' siguen poniendo a las ciencias de lo social dentro del estatuto vago, pero desvalorizante, de ciencias 'blandas"'? (Lahire, 2006, p. 365). Si bien es mucho el camino que todavía queda por trazar, tal vez sea hora de reconocer que hemos avanzado algunos peldaños y empezar a pensarnos desde allí.

\section{CONClusiones: ¿SOCIOLOGÍA PARA PROMOVER EL “PENSAMIENTO SOCIAL”?}

En el presente trabajo se intentó hacer un recorrido por la forma de enseñanza de la sociología en el nivel medio en tres países del Cono Sur. Para ello también se recuperó, de forma sintética, parte de la historia de la carrera de Sociología y su tradición académica en el ámbito universitario. Asimismo, se revisaron DC de la asignatura, manuales y otras normativas referidas al sistema educativo. El recorte elegido, focalizando en la realidad de estos tres países desde un enfoque comparativo (Piovani y Krawczyk, 2017), responde a la necesidad de analizar en conjunto de experiencias que presentan entre sí puntos en común, así como notables diferencias en el ámbito educativo (Ruiz y Schoo, 2014). Como ya se mencionó, presentar una mirada generalizada de estos tres casos supone valorar no sólo los alcances de este tipo de estudios sino también advertir sobre sus limitaciones. En ese sentido, se considera que el presente trabajo puede ser un punto de partida para investigaciones futuras que se propongan indagar respecto de esta temática al interior de cada territorio nacional, mostrando las especificidades y las diferencias regionales dentro de cada país.

A modo de cierre, es interesante destacar la particularidad del caso brasilero y el temprano desarrollo de cursos de enseñanza de Sociología en el nivel medio. En ese marco y en relación a lo mencionado en el párrafo precedente, no podemos perder de vista que estas experiencias se dan en un territorio caracterizado por una 
enorme desigualdad social y económica, situación que incide especialmente al pensar el sistema educativo. Uruguay y también Argentina (pese a sus enormes diferencias) presentan un panorama algo más equitativo en materia educativa, no sólo en términos históricos, sino también por la implementación de las leyes de 2006 y 2008, respectivamente. Inclusive si consideramos que el establecimiento de la obligatoriedad del nivel medio no trajo aún diferencias significativas entre tasas de ingreso y promoción en Argentina y Uruguay (Terigi, Briscioli, Scavino, Morroney Toscano, 2013; De Armas y Retamoso, 2010), la disparidad entre estas tasas continúa siendo mayor en Brasil de acuerdo con un estudio de Ruiz y Schoo (2014),quienes analizan los impactos de la ampliación de la obligatoriedad escolar en cinco países de la región.

Pensar en la especificidad de la enseñanza de la sociología como asignatura escolar implica no olvidar el contexto en el cual se dicta. Su enseñanza no puede pensarse de forma escindida con el resto del contexto educativo y es por ello que se ha hecho una sucinta revisión no sólo de la asignatura foco de este trabajo, sino también de otros datos relativos, especialmente, a la educación media.

Como se dijo en una de las primeras notas al pie, originalmente, el recorte se centró sobre cuatro casos. La decisión de excluir del análisis la experiencia chilena respondió a que en aquel país no se dicta una asignatura bajo el nombre de "Sociología". Sin embargo, al recuperar el programa de estudio del bloque de Historia, Geografía y Ciencias Sociales que se imparte en Chile en los últimos dos años del nivel medio para la orientación "Humanístico científica", es posible reconocer puntos de conexión entre esta normativa y los DC de Sociología de Argentina, Uruguay y Brasil. En este sentido, el denominador común no se encuentra tanto en las temáticas impartidas sino en cierta disposición en el vínculo con el aprendizaje y el conocimiento. Es así como encontramos una apuesta por "un foco multidisciplinario permanente" que involucra allí saberes de distintas disciplinas, entre ellas la sociología (Programa de estudio Chile, 2015, p. 32), asentado sobre una experiencia previa, desde los primeros años del nivel medio, que promueve desde el mismo bloque curricular el desarrollo del "pensamiento crítico"(Bases Curriculares Chile, 2016, p.185).

En consecuencia, tal vez, lo más disruptivo que puede ofrecernos la sociología en el nivel medio no es tanto (o no es sólo) su especificidad "disciplinar": su enfoque en ciertos autores, temáticas y perspectivas; sino una determinada forma de mirar el mundo y (tratar de) comprenderlo (y por qué no de transformarlo). Una mirada crítica, desnaturalizadora, incómoda, que tiene que ver con el desarrollo de este "pensamiento social" al que refiere Pipkin y que puede trabajarse no sólo en clases de Sociología sino también desde otras asignaturas afines (como las que conforman las áreas de Ciudadanía, en el caso argentino, o el bloque de Educación Social y Cívica, en Uruguay). La "utopía realista" por la que aboga Lahire (2006) no debería agotarse en una demanda por más ciencias sociales en las escuelas sino también por más docentes (y mejores) docentes de Sociología en estas disciplinas.

\section{Bibliografía}

Actis, C., Cuestas, P., Di Bastiano, R., Fux, J., Mendez Isla, M., Molinari, V. y Sosa, N. (2018). Sociología y educación secundaria: reflexiones en torno a los contenidos del DC de Sociología de la Prov. de Buenos Aires. X Jornadas de Sociología de la UNLP, 5 al 7 de diciembre de 2018, Ensenada, Argentina. En: [Actas]. Ensenada: Universidad Nacional de La Plata. Facultad de Humanidades y Ciencias de la Educación. Recuperado de: http://www.mem oria.fahce.unlp.edu.ar/trab_eventos/ev.11329/ev.11329.pdf

Bethel, L. (1992). Historia de América Latina(tomo 10). Barcelona: Editorial Crítica.

Blois, P. (2015). La institucionalización y profesionalización de la Sociología en Brasil y Argentina. Formación, organización e intervención de los sociólogos. Estudios sociológicos de El Colegio de México, 33(99).Recuperado de:https://estudiossociologicos.colmex.mx/index.php/es/article/view/1393/1554

Bourdieu, P. (1990). Una ciencia que incomoda. En P. Bourdieu, Sociología y cultura. México: Grijalbo.

Chevallard, Y. (1991). La transposición didáctica. Del saber sabio al saber enseñado. Buenos Aires: Aique. 
De Armas, G. y Retamoso, A. (2010). La universalización de la educación media en Uruguay. Tendencias, asignaturas pendientes y retos a futuro. Montevideo: Unicef.

De Cesare, M. (2006).Practicing Public Sociology in the Classroom: Teaching Sociology in High School. Draft for a presentation at California State University, Northridge. Recuperado de: http://www.csun.edu/ csbs/events/p resentations/decesare.pdf

Lahire, B. (2006). Hacia una utopía realista: enseñar las ciencias del mundo social desde la escuela primaria. En B. Lahire, El espiritu sociológico. Buenos Aires: Manantial.

Marrero, A. (2010). Formación docente y producción de conocimiento en el sistema de educación superior uruguayo: una cuestión sin resolver. Revista Argentina de Educación Superior, 2(2). Recuperado de: file://C:/Users/Cimecs/Downloads/DialnetFormacionDocenteYProduccionDeConocimientoEnElSiste-6488175\%20(1).pdf

Mignolo, W. (2007). La idea de América Latina. La herida colonial y la opción decolonial. Barcelona: Gedisa.

Molinari, V. (2009). La enseñanza de la Sociología en el nivel medio: Una mirada desde los practicantes de Sociología. Cuestiones de Sociologia,5-6, 391-405. Recuperado de: http://sedici.unlp.edu.ar/bitstream/handle/10915/1315 5/Documento_completo.pdf?sequence=1\&isAllowed $=\mathrm{y}$

Motta de Souza Martínez, D. R. (2016). La sociología como disciplina escolar en el Uruguay. Estudio focalizado en Educación Secundaria y Formación Docente entre 1963 y 2008(Tesis de Maestría en política y gestión de la Educación).Instituto Universitario CLAEH, Montevideo.

Moura de Oliveira, G., yVeríssimoVeronese, M. (2019). Brasil y el"fenómeno Bolsonaro": un análisis preliminar. Revista Mexicana de Ciencias Politicas y Sociales, 64(237), 245-268. Recuperado de: https://www.ssoar.info/ssoar/bitstream/handle/document/64056/ssoar-rmcpys-2019-237-moura_de_oli veira_et_al-Brasil_y_el_fenomeno_Bolsonaro.pdf?sequence=1\&isAllowed=y\&lnkname=ssoar-rmcpys-2019237-moura_de_oliveira_et_al-Brasil_y_el_fenomeno_Bolsonaro.pdf

Oliveira, A. (2013). O currículo de Sociología na escola: um campo em construçao (e disputa). Espaço do currículo, 6(2), 355-366. Recuperado de: https://periodicos.ufpb.br/index.php/rec/article/view/15308/9756

Oliveira, A.(2015). Um balanço sobre o campo do ensino de sociologia no Brasil. Em Tesse, 12(2), 6-16. Recuperado de: file://C:/Users/Cimecs/Downloads/41691-140498-1-PB.pdf

Pereyra, D. y Pontremoli, C. (2014). ¿La Sociología está pasada de moda? Una discusión sobre la enseñanza de Sociología en la escuela media en Argentina: docentes, estudiantes y propuesta curricular. Educaçao y Realidade, Porto Alegre, 39(1), 139-159. Recuperado de: http://www.scielo.br/scielo.php?script=sci_arttext\&pid=S2175 $-62362014000100009 \& \operatorname{lng}=\mathrm{pt} \& \mathrm{nrm}=\mathrm{iso} \& \mathrm{t} \operatorname{lng}=\mathrm{es}$

Pipkin, D. (comp.) (2009). Pensar lo social. Un aporte de la enseñanza de la Sociología para la escuela media. Buenos Aires: La Crujía.

Piovani, J. I. y Krawczyk, N. (2017). Los estudios comparativos: algunas notas históricas, epistemológicas y metodológicas. Educaçao y Realidade, Porto Alegre, 42(3), 821-840. Recuperado de: https://www.scielo.br/pdf /edreal/v42n3/2175-6236-edreal-42-03-00821.pdf

Presedo, C. (2017). La identidad profesional de los docentes en educación media según su formación terciaria: que impacto tiene esta en su concepción del rol del profesor (Tesina de grado de la Licenciatura en Sociología).Universidad de la República, Montevideo.

Rama, G. (2004). La evolución de la educación secundaria en Uruguay. REICE, Revista Electrónica Iberoamericana sobre calidad, eficacia y cambio en educación, 2(1). Recuperado de: https://revistas.uam.es/index.php/reice/artic le/view/5533/5951

Ruiz, M. C. y Schoo, S. (2014). La obligatoriedad de la educación secundaria en América Latina. Convergencias y divergencias en cinco países. Foro de Educación, 12(16), 71-98. Recuperado de: file://C:/Users/Cimecs/ Downloads/Dialnet-LaObligatoriedadDeLaEducacionSecundariaEnAmericaLa-4684139.pdf

Sabatovich Fernández, D. (2018). La enseñanza de la Sociología: entre contradicciones y armonias(Tesis de Maestría en Educación, Sociedad y Política).Facultad Latinoamericana de Ciencias Sociales - Programa Uruguay, Montevideo. 
Santos, M. B. (2012). Diretrizes curriculares estaduais para o ensino de sociología: em busca do mapa comum. PerCursos, 13(1), 40-59. Recuperado de: http://www.revistas.udesc.br/index.php/percursos/article/view/243 $9 / 2066$

Suasnábar, C. y Rovelli, L. (2010). Gobierno y reformas de la educación en la Argentina reciente. Revue international d'éducation de Sèvres. Sèvres: Centre internationald'étudespédagogiques, 55, 17-26. Recuperado de: https://sistemaeducativouna.files.wordpress.com/2014/10/gobierno-y-reformas-de-la-educacic3b3n-en-laargentina-reciente.pdf

Tedesco, J. C. (1993). Educación y Sociedad en la Argentina (1880-1945). Buenos Aires: Ediciones Solar.

Terigi, F., Briscioli, B., Scavino, C., Morrone, A., yToscano, A. G. (2013). La educación secundaria obligatoria en la Argentina: entre la expansión del modelo tradicional y las alternativas de baja escala. Revista del IICE, 33, 27-46. Recuperado de: http://revistascientificas.filo.uba.ar/index.php/iice/article/view/1099/1078

\section{Normativa}

Argentina. Ministerio de Educación (2011). Diseño Curricular de Sociología para la Provincia de Buenos Aires. Recuperado de: https://www.abc.gov.ar

Argentina. Ministerio de Educación (2009). Resolución del Consejo Federal de Educación. Recuperado de: https://cfe .educacion.gob.ar/resoluciones/res09/84-09-anexo01.pdf

Chile. Ministerio de Educación de Chile (2016).Bases Curriculares, $7^{\circ}$ básico a $2^{\circ}$ medio. Recuperado de: https://ww w.curriculumnacional.cl/614/articles-37136_bases.pdf

Chile. Ministerio de Educación de Chile (2015). Programa de Estudio Cuarto medio: Historia, Geografía y Ciencias Sociales. Recuperado de: https://www.curriculumnacional.cl/614/articles-34443_programa.pdf

Uruguay. Ministerio de Educación (2006). Programa de Sociología de Uruguay Recuperadode: https://www.ces.edu.uy/files/Planes\%20y\%20programas/Ref\%202006\%20Bach/5humanistic o/social.pdf?fbclid=IwAR13EuwkZgr4j2yGeQwFjrsE4HpRxBqDXVwEkzSGcw_3qS5DDEPx2MGuqRY

\section{Notas}

1 Agradezco la atenta lectura de mi colega Victoria Molinari a una versión preliminar de este trabajo. Sus comentarios a aquel borrador fueron de suma importancia para enriquecer este nuevo texto, tanto como los de quien evaluó la primera versión de este artículo durante su proceso de revisión. Asimismo, quiero agradecer a amigas/os, docentes y colegas uruguayos/as, chilenos/as y brasileros/assin cuya guía y experiencia este trabajo no hubiera sido posible: Sebastián Muñoz Tapia, Antonino Opazo Beaza, Sofia Vanoli, Evangelina Sosa, Joanna Peluffo Velarde, Iñaki Amilivia Kunin, Adriana Marrero, Diego Piñeiro, María del Lujan Peppe, Dinorah Motta de Souza y Amurabi Oliveira.

2 Esta afirmación se basa en una actividad que llevamos adelante en el marco de la cátedra "Didáctica especial y prácticas de la enseñanza en Sociología y Ciencias Sociales” del Profesorado en sociología, de la Facultad de Humanidades y Ciencias de la Educación de la Universidad Nacional de la Plata (UNLP). En los primeros encuentros se les solicita a las/os estudiantes (futuras/os practicantes en escuelas del nivel medio) que escriban un ensayo crítico a partir de esa pregunta.

3 En un primer momento el recorte se pensó a través del análisis de los casos argentino, uruguayo, brasilero y chileno. El porqué de esta decisión respondía a la potencial riqueza de un estudio comparativo entre estos cuatro países, por los muchos puntos en común que hacen a nuestra historia regional (Bethel, 1992; Mignolo, 2007). Sin embargo, al comenzar a realizar esta investigación constaté, a través de fuentes primarias (las/os informantes mencionados en los agradecimientos previos), que en Chile no existía una asignatura llamada "Sociología” en el nivel medio. No obstante, en las conclusiones de este trabajo se retomarán, brevemente, algunos modos en que la disciplina puede "inmiscuirse" en las aulas escolares pese a no contar con un espacio curricular específico.

4 Una resolución del Consejo Federal de Educación (disponible en: https://cfe.educacion.gob.ar/resoluciones/res09/84 -09-anexo01.pdf) establece en su artículo 63 que "Para cada Orientación, cada Jurisdicción definirá un único plan de formación, que tendrá alcance provincial. En el mismo se explicitarán márgenes y criterios jurisdiccionales de definición curricular institucional". Esta fue la normativa de base para la formulación de este DC.

5 Se encuentran disponibles online los DC y lineamientos básicos de las provincias de Santa Fe, Entre Ríos, Tucumán, Mendoza, Río Negro, Córdoba. 
6 Esto no implica que su utilización sea privativa del territorio bonaerense, solo que la experiencia empírica que da sustento a esta información no ha podido ser comprobada en otras provincias.

7 Sobre las influencias que la epistemología del Derecho pudo tener respecto de la enseñanza de la sociología en Uruguay, en particular, se recomienda la lectura de Sabatovich Fernández (2018).

8 Mencionamos únicamente el nivel universitario ya que un análisis de la carrera de Sociología a nivel terciario en los tres países implicaría un trabajo mucho mayor que excede ampliamente nuestros objetivos.

9 Cabe aclarar que en la mayoría de las Universidades Nacionales en las que se dicta la carrera de Profesorado en Sociología, esta se plantea como una instancia optativa posterior a la Licenciatura y para la cual es requisito contar con el título de licenciado/a.

10 Es preciso destacar el carácter todavía “joven” de la tradición sociológica ya que permite explicar, en parte, lo que vemos en las aulas escolares: que la mayor parte de quienes están a cargo de la asignatura "Sociología” no son de hecho profesoras/es en esta disciplina. Una situación similar reconoce Presedo (2017) para el caso uruguayo y analiza Sabatovich Fernández (2018) en clave histórica. 